\title{
Elliptic Root System and Elliptic Artin Group
}

By

\author{
Hiroshi Y $\mathrm{AMADA}^{*}$
}

\section{$\S 1$. Introduction}

The concepts of elliptic root system, elliptic Dynkin diagram and elliptic Weyl group were introduced by K. Saito to describe the Milnor lattices and the flat structures of semi-universal deformations for simply elliptic singularities [S], [S0], [S1], [S2].

Generators and relations of elliptic Weyl groups were studied in the context of elliptic Dynkin diagrams by K. Saito and T. Takebayashi [ST]. (This presentation of an elliptic Weyl group is a generalization of a Coxeter system. See Theorem 2.1). In their paper, they proposed the following problem: find generators and relations of "elliptic Lie algebras", "elliptic Hecke algebras" and "elliptic Artin groups" (the fundamental groups of the complements of the discriminant for simply elliptic singularities) in terms of the elliptic Dynkin diagrams.

In [SY], applying R. Borcherd's construction of vertex algebras [Borl], [Bor2], K. Saito and D. Yoshii constructed the elliptic Lie algebras (which are isomorphic to the toroidal algebras [MEY]) for homogeneous elliptic Dynkin diagrams. In addition, they described the fundamental relations in terms of the generators attached to the elliptic Dynkin diagrams. These relations are a generalization of the Serre type relations (for other appraches cf. $[\mathrm{BM}]$, [S11]).

In this article, we shall give an answer to their problem for the case of elliptic Artin groups and elliptic Hecke algebras as an application of the twisted Picard-Lefschetz formula due to A. B. Givental [Gi]. As for the former groups, they have already been studied by $\mathrm{H}$. van der Lek

Communicated by K. Saito, August 6, 1999. Revised August 27, 1999.

1991 Mathematics Subject Classification(s): 14D05

This research was supported by Grant-in-Aid for Scientific Research (No. 10640054),

Ministry of Education, Science, Sports and Culture of Japan.

* Kitami Institute of Technology, Kitami, Hokkaido 090, Japan 
[L] under the name of extended Artin groups and from the view point of affine Dynkin diagrams for arbitrary affine root systems (see Theorem 5.1). Here we describe these groups in term of generators associated to the vertices of elliptic Dynkin diagrams that reflect the geometry of vanishing cycles of simply elliptic singularities (Theorem 5.2). For this purpose, we restrict ourselves to the 1-codimensional case (Definition 2.3) that has rich geometry such as flat structure. As for the latter algebras, which are subalgebras of Cherednik's double affine Hecke algebras [C1], [C2], [C3], we can construct some irreducible finite dimensional representations as monodromy representations (Proposition 6.1).

The present paper is organized as follows. In Sect. 2, we give a brief review of elliptic root systems and elliptic Weyl groups. In Sect. 3, we will explain A. B. Givental's twisted Picard-Lefschetz formula. Then we will apply his theory to the case of simply elliptic singularites (Proposition 3.1). In Sect. 4, we will define elliptic Artin groups (Definition 4.1) and explain the relation between elliptic Weyl goups and elliptic Artin groups (Theorem 4.1). In Sect. 5, we will explain the result of $\mathrm{H}$. van der Lek about extended Artin groups (Theorem 5.1). Then we shall compare the Artin groups with extended Artin groups (Theorem 5.2). In Sect. 6, we shall define elliptic Hecke algebras, and then we shall obtain finite dimensional irreducible representations of them (Proposition 6.1).

The author would like to express his appreciation to Kyoji Saito, Peter Slodowy and Norio Suzuki for valuable discussions and useful comments. He also thanks Kenji Iohara and Endo Kobayashi for kind advice.

\section{§ 2. Elliptic Root Systems and Elliptic Weyl Groups}

We briefly explain elliptic root systems and elliptic Weyl groups following [S1]. Let $F$ be a vector space over $\mathbb{R}$ with symmetric bilinear form $(\cdot, \cdot)$ of signature $\left(l_{+}, l_{0}, l_{-}\right)$, where $l_{+}\left(\right.$resp. $\left.l_{-}\right)$is the dimension of a maximal positive (resp. negative) definite subspace of $F$ and $l_{0}$ is the dimension of the radical of $\left(\cdot,{ }^{\circ}\right)$. For $\alpha \in F$ such that $(\alpha, \alpha) \neq 0$, we define

$$
\begin{gathered}
\alpha^{\vee}:=\frac{2}{(\alpha, \alpha)} \alpha, \\
w_{\alpha}(u):=u-\left(u, \alpha^{\vee}\right) \alpha \text { for any } u \in F .
\end{gathered}
$$


Definition 2.1. A subset $R \subset F$ is called an elliptic root system, if the following conditions are satisfied:

(R. 1) $\left(l_{+}, l_{0}, l_{-}\right)=(l, 2,0)$.

(R. 2) Let $Q(R)$ be the $\mathbf{Z}$-submodule of $F$ generated by $R$. Then, $Q(R) \otimes_{\mathbf{Z}} \mathbf{R}$ $=F$.

(R. 3) For any $\alpha \in R,(\alpha, \alpha) \neq 0$.

(R.4) $w_{\alpha}(R)=R$.

(R. 5) $\left(\alpha, \beta^{\vee}\right) \in \mathbf{Z}$ for any $\alpha, \beta \in R$.

(R. 6) $R$ is irreducible, i.e. if $R=R_{1} \cup R_{2}$ with $R_{i}$ satisfying $(R, 1) \sim$ $(R, 5)$ and $R_{1} \perp R_{2}$, then $R_{1}=\phi$ or $R_{2}=\phi$.

We call the Weyl group $W(R):=\left\langle w_{\alpha} \mid \alpha \in R\right\rangle$ associated with $R$ elliptic Weyl group of $R$. Also K. Saito defined an elliptic Dynkin diagram for an elliptic root system as follows: Let $G$ be a 1-dimensional subspace of $\operatorname{rad}(\cdot, \cdot)$ which is defined over $\mathbf{Q}$ in the sense of K. Saito (see [S1]). Then, the image of $R$ in $F / G$ is an affine root system $R_{a}$. We fix a generator a of the lattice $G \cap \operatorname{rad}(\cdot, \cdot)$. Note that the generator $a$ is unique up to a choice of sign. We call $(R, G)$ the marked elliptic root system. For any $\alpha \in R_{a}$, put

$$
k(\alpha):=\inf \left\{k \in \mathbf{Z}_{>0} \mid \alpha+k \cdot a \in R\right\}
$$

and

$$
\alpha^{*}:=\alpha+k(\alpha) \cdot a
$$

$k(\alpha)$ is called the counting of $\alpha$. The following proposition is known.

Proposition 2.1 (K. Saito $[\mathrm{S} 1])$. Let $(R, G)$ be a marked elliptic root system. Then we have

$$
R=\left\{\alpha+m \cdot k(\alpha) \cdot a \mid \alpha \in R_{a}, m \in Z\right\} .
$$

Let $\Gamma\left(R_{a}\right)=\left\{\alpha_{0}, \alpha_{1}, \ldots, \alpha_{l}\right\}$ be a basis of $R_{a}$ such that $\left\{\alpha_{1}, \ldots, \alpha_{l}\right\}$ form the simple roots of an underlying finite root system, and let $b:=\sum_{i=0}^{l} n_{i} \alpha_{i}$ be the minimal imaginary root of $R_{a}$. The set of exponents of $(R, G)$ is 


$$
\left\{0, m_{\alpha}:=\frac{(\alpha, \alpha)_{R}}{2 \cdot k(\alpha)} \cdot n_{\alpha} \mid \alpha \in \Gamma\left(R_{a}\right)\right\}
$$

where $(\cdot,)_{R}$ is a constant multiple of $(\cdot, \cdot)$ normalized in the way that inf $\left\{(\alpha, \alpha)_{R} \mid \alpha \in R\right\}$ is equal to 2 . Set

$$
\Gamma\left(R_{a}\right)_{\max }:=\left\{\alpha \in \Gamma\left(R_{a}\right) \mid m_{\alpha}=\max \left\{m_{\beta} \mid \beta \in \Gamma\left(R_{a}\right)\right\}\right\}
$$

and

$$
\Gamma\left(R_{a}\right)_{\max }^{*}:=\left\{\alpha^{*} \mid \alpha \in \Gamma\left(R_{a}\right)_{\max }\right\}
$$

Definition 2.2. The elliptic Dynkin diagram $\Gamma(R, G)$ of the marked elliptic root system $(R, G)$ is a finite graph generated by the set of vertices $\Gamma(R, G):=\Gamma\left(R_{a}\right) \cup \Gamma\left(R_{a}\right)_{\max }^{*}$ and connected according to the following conditions: for $\alpha, \beta \in \Gamma(R, G)$

(1) $\left(\alpha, \beta^{\vee}\right)=0$

$\alpha \bigcirc \quad \bigcirc \beta$

(2) $\left(\alpha, \beta^{\vee}\right)=\left(\alpha^{\vee}, \beta\right)=-1$

$\alpha \bigcirc-\bigcirc \beta$

(3) $\left(\alpha, \beta^{\vee}\right)=-t,\left(\alpha^{\vee}, \beta\right)=-1$ for $t=2,3,4$

$\alpha \bigcirc \rightarrow$

(4) $\left(\alpha, \beta^{\vee}\right)=\left(\alpha^{\vee}, \beta\right)=2$

$\alpha \mathrm{O}=--=\mathrm{O} \beta$

Here and afterwards we shall use the convention:

$$
\mathrm{O}-\mathrm{O}=\mathrm{O} \underset{t}{\rightarrow} \mathrm{O}=\mathrm{O} \underset{t}{\leftarrow} \mathrm{O} \quad \text { for } t=1
$$

and

$$
\mathrm{O} \underset{t}{\rightarrow} \mathrm{O}=0 \underset{t^{-1}}{\leftarrow} \quad \text { for } t=2^{ \pm 1}, 3^{ \pm 1} \text { and } 4^{ \pm 1}
$$

Definition 2.3. An elliptic Dynkin diagram $\Gamma(R, G)$ is called 1codimensional if the number of vertices of $\Gamma\left(R_{a}\right)_{\max }$ is equal to 1 except $A_{1}^{(1,1) *}$.

Remark 2.1. The class of 1-codimensional elliptic Dynkin diagrams is an important subclass of elliptic Dynkin diagrams. Indeed, this is the only case that a flat structure has been constructed in [S2].

From now on we shall assume that the elliptic Dynkin diagram is 1-codimensional (see Table 1). 
To state the structure theorem of elliptic Weyl groups, we explain foldings of elliptic Dynkin diagrams due to K. Saito [S1].

Let $(R, G)$ be a 1-codimensional marked elliptic root system in a vector space over $\mathbf{R}$, say $F$, with a symmetric bilinear form $(\cdot, \cdot)$ and $R_{a}$ an affine root system which is the image of $R$ in $F / G$. Set

$$
\operatorname{Aut}(R, \operatorname{rad}(\cdot, \cdot)):=\left\{f \in \operatorname{Aut}(F,(\cdot, \cdot))|f(R)=R, f|_{\operatorname{rad}(\cdot, \cdot)}=i d_{\operatorname{rad}(\cdot, \cdot)}\right\} .
$$

Then there exists the natural projection

$$
\operatorname{Aut}(\Gamma(R, G)) \rightarrow \operatorname{Aut}\left(\Gamma\left(R_{a}\right)\right)
$$

and a section $\phi$

$$
\operatorname{Aut}\left(\Gamma\left(R_{a}\right)\right) \rightarrow \operatorname{Aut}(R, \operatorname{rad}(\cdot, \cdot))
$$

where $\operatorname{Aut}(\Gamma(R, G))$ (resp. $\operatorname{Aut}\left(\Gamma\left(R_{a}\right)\right)$ ). is the group of automorphisms of the elliptic Dynkin diagram $\Gamma(R, G)$ (resp. affine Dynkin diagram $\left.\Gamma\left(R_{a}\right)\right)$. Then we have

$$
\operatorname{Aut}\left(\Gamma\left(R_{a}\right)\right) \cong \operatorname{Aut}(\Gamma(R, G)) / Z_{2}
$$

where $Z_{2}$ is the group generated by the parmutation of $\alpha$ and $\alpha^{*}$ for $\alpha \in \Gamma\left(R_{a}\right)_{\max }$.

Let $H$ be a subgroup of $\operatorname{Aut}\left(\Gamma\left(R_{a}\right)\right)$ which acts on $F$ through the section $\phi$. Let $F^{H}$ be the invariant subspace of $F$ by $H$. We define two mappings as follows:

$$
\begin{array}{rlrl}
\operatorname{Tr}^{H}: F & \rightarrow F^{H}, & x \mapsto \sum_{y \in H x} y \\
\operatorname{Tr}_{H}: F \rightarrow F^{H}, & x \mapsto(\#\{H x\})^{-1} \sum_{y \in H x} y
\end{array}
$$

where \#ignifies cardinality. Then we obtain the following lemma:

Lemma 2.1 (K. Saito [S1] Section 12). There exist marked elliptic root systems $\left(R^{H}, G\right)$ and $\left(R_{H}, G\right)$ in $F^{H}$ with the symmetric bilinear form $\left.(\cdot, \cdot)\right|_{F^{H}}$ such that the set of vertices of $\operatorname{Tr}^{H}(\Gamma(R, G))$ and $\operatorname{Tr}_{H}(\Gamma(R, G))$ form elliptic Dynkin diagrams for $\left(R^{H}, G\right)$ and $\left(R_{H}, G\right)$ respectively.

The above procedures which give $\left(R^{H}, G\right)$ and $\left(R_{H}, G\right)$ are called folding and mean folding respectively. We list $\left(R^{H}, G\right)$ and $\left(R_{H}, G\right)$ in the following table: 


\begin{tabular}{|c|c|c|c|c|c|}
\hline$(R, G)$ & $D_{4}^{(1,1)}$ & $D_{4}^{(1,1)}$ & $E_{6}^{(1,1)}$ & $E_{6}^{(1,1)}$ & $E_{7}^{(1,1)}$ \\
\hline$H$ & $Z_{2} \times Z_{2}$ & $Z_{3}$ & $Z_{3}$ & $Z_{2}$ & $Z_{2}$ \\
\hline$\left(R^{H}, G\right)$ & $B C_{1}^{(2,4)}$ & $G_{2}^{(3,3)}$ & $G_{2}^{(1,3)}$ & $F_{4}^{(2,2)}$ & $F_{4}^{(1,2)}$ \\
\hline$\left(R_{H}, G\right)$ & $B C_{1}^{(2,1)}$ & $G_{2}^{(1,1)}$ & $G_{2}^{(3,1)}$ & $F_{4}^{(1,1)}$ & $F_{4}^{(2,1)}$ \\
\hline
\end{tabular}

The list of 1-codimensional elliptic Dynkin diagram is given in the appendix.

Now we attach an affine root system $(R, G)_{a}$ to a marked elliptic root system $(R, G)$ as follows:

$$
(R, G)_{a}= \begin{cases}R_{a} & \text { if } R_{a}=R_{a}^{\vee} \\ R_{a} & \text { if }(R, G) \text { is obtained by folding } \\ R_{a}^{\vee} & \text { if }(R, G) \text { is obtained by a mean folding }\end{cases}
$$

where $R_{a}$ is given as before.

Next we introduce $\tilde{t}_{\alpha} \in \tilde{W}(R, G)$ for all $\alpha \in \Gamma\left(R_{a}\right)$ as follows: For $\alpha_{0} \in \Gamma\left(R_{a}\right)_{\max }$, set

$$
\tilde{t}_{\alpha_{0}}:=r_{\alpha_{0}} r_{\alpha_{0}}{ }^{*}
$$

If $\alpha_{1}, \ldots, \alpha_{k} \in \Gamma\left(R_{a}\right) \backslash\left\{\alpha \mid \alpha \in \Gamma_{a, \text { max }}\right\}$ are arranged in the following position:

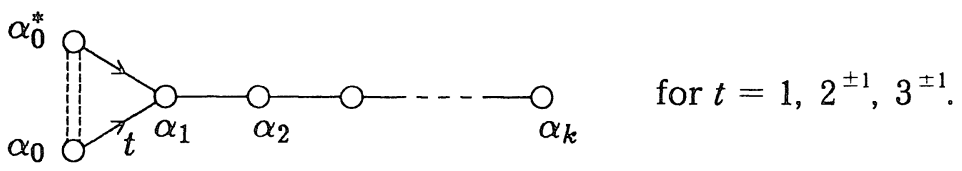

Then we define

$$
\tilde{t}_{\alpha_{j+1}}:=r_{\alpha_{j+1}} \tilde{t}_{\alpha_{j}} r_{\alpha_{j+1}} \tilde{t}_{\alpha_{j}}^{-1}
$$

inductively.

Let us define the number

$$
m(R, G):=\frac{\max \left\{m_{\alpha} \mid \alpha \in \Gamma\left(R_{a}\right)\right\}}{\operatorname{gcd}\left\{m_{\alpha} \mid \alpha \in \Gamma\left(R_{a}\right)\right\}}
$$

which plays the role of the Coxeter number for the elliptic root system 
[S0], [S1], [S2], [ST].

K. Saito and T. Takebayashi [ST] gave presentations of elliptic Weyl groups as follows:

Theorem 2.1 (K. Saito and T. Takebayashi [ST]). Let $(R, G)$ be a marked elliptic root system and $\tilde{W}(R, G)$ the group defined by the following generators and relations:

generators: $r_{\alpha}$

relations:

$$
\alpha \in \Gamma(R, G)
$$

(W.0) $\quad r_{\alpha}^{2}=1$

(W.1.0) $\quad\left(r_{\alpha} r_{\beta}\right)^{2}=1$

(W.1.1) $\quad\left(r_{\alpha} r_{\beta}\right)^{3}=1$

(W.1.2) $\quad\left(r_{\alpha} r_{\beta}\right)^{4}=1$

(W.1.3) $\quad\left(r_{\alpha} r_{\beta}\right)^{6}=1$

(W.2.1) $\quad\left(r_{\alpha} r_{\beta} r_{\alpha}, r_{\beta}\right)^{3}=1$

(W.2.2) $\quad\left(r_{\alpha} r_{\beta} r_{\alpha} \cdot r_{\beta}\right)^{2}=1$

(W.2.3) $\quad\left(r_{\alpha} r_{\beta} r_{\alpha}, r_{\beta}\right)^{3}=1$ and $\left(r_{\alpha} r_{\beta} r_{\alpha} \cdot r_{\beta} r_{\alpha} r_{\beta}\right)^{2}=1$

(W.2.4) $\quad\left(r_{\alpha} r_{\alpha}, r_{\beta}\right)^{2}=\left(r_{\alpha}, r_{\beta} r_{\alpha}\right)^{2}=\left(r_{\beta} r_{\alpha} r_{\alpha}\right)^{2}$

(W.3) $\quad\left(r_{\alpha} r_{\beta} r_{\alpha} r_{\beta} r_{\gamma} r_{\beta}\right)^{2}=1$ and $\left(r_{\alpha} r_{\beta}^{*} r_{\alpha} r_{\beta} r_{\gamma} r_{\beta}\right)^{2}=1$ for $t=1,2^{ \pm 1}, 3^{ \pm 1}$ $\alpha \circ$

$\alpha \circ \circ \beta$

$\alpha \circ \longrightarrow \beta$

$\alpha \underset{2^{ \pm 1}}{\longrightarrow} \mathrm{\bigcirc}$

$\alpha \underset{3^{ \pm 1}}{\longrightarrow} \beta$

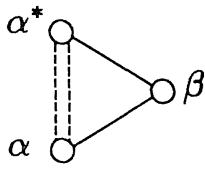

$\sum_{\alpha} \beta$
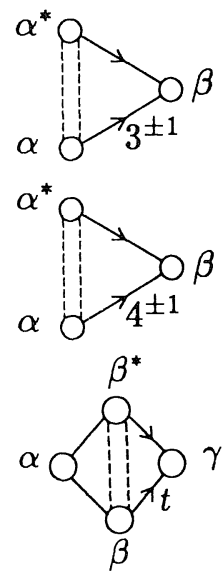
where the two relations in (W.3) are equivalent in the case of $t=1$.

(i) Let $\tilde{N}(R, G)$ be the subgroup of $\tilde{W}(R, G)$ generated by $\left\{\tilde{t}_{\alpha} \mid \alpha \in\right.$ $\Gamma(R, G)\}$. Then $\tilde{N}(R, G)$ is a free abelian subgroup isomorphic to the lattice $Q\left((R, G)_{a}\right)$.

(ii) Set $\tilde{c}(R, G):=\prod_{\alpha \in \Gamma(R, G) \backslash \Gamma\left(R_{a}\right)_{\max }} r_{\alpha} \prod_{\alpha \in \Gamma\left(R_{a}\right)_{\max }} r_{\alpha} r_{\alpha^{*}}, \quad$ then its power $\tilde{c}(R, G)^{m(R, G)}$ generates the center of $\tilde{W}(R, G)$ and belongs to $N(R, G)$. In particular, $\tilde{c}(R, G)^{m(R, G)}$ is expressed as

$$
\tilde{c}(R, G)^{m(R, G)}=\prod_{\alpha \in \Gamma\left(R_{a}\right)} \tilde{t}_{\alpha}^{n_{a}}
$$

where the $n_{\alpha}$ are the coefficients of the generator $b$ of the imaginary roots in $(R, G)_{a}$.

(iii) The quotient group of $\tilde{W}(R, G)$ by the center $\left\langle\tilde{c}(R, G)^{m(R, G)}\right\rangle$ is isomorphic to the elliptic Weyl group $W(R)$ :

$$
\tilde{W}(R, G) /\left\langle\tilde{c}(R, G)^{m(R, G)}\right\rangle \cong W(R) .
$$

Here the relations $(W .1 .0) \sim(W .1 .3)$ are well known as Coxeter relations, and the relations $(W .2 .1) \sim(W .3)$ are new ones due to the broken bonds in the diagram. Let us call them elliptic Coxeter relations and the group $\tilde{W}(R, G)$ hyperbolic extension of elliptic Weyl group of $W(R)$ (see $[\mathrm{S} 1],[\mathrm{ST}])$.

Remark 2.2. In this article, we only treat 1-codimensional elliptic Dynkin diagrams. In [ST], K. Saito and T. Takebayashi treated more general elliptic root systems.

\section{§ 3. Twisted Picard-Lefschetz Formula}

The relations of elliptic Weyl groups were obtained by studying the monodromy representations of simply elliptic singularities. To find generators and their relations of elliptic Artin groups attached to elliptic Dynkin diagrams, we want to construct a certain kind of deformation of the monodromy representations of simply elliptic singularities. Fortunately, A. B. Givental [Gi] has already studied q-deformation of monodromy representations of isolated hypersurface singularities using the so-called twisted Picard-Lefschetz formula (see also F. Pham [P] and I. Shimada [Sh]).

First, we explain the classical Picard-Lefschetz formula. 
Let $f:\left(\mathbf{C}^{3}, 0\right) \rightarrow(\mathbf{C}, 0)$ be a polynomial mapping such that $f^{-1}(0)$ has a simply elliptic singularity (see $[\mathrm{S}]$ ). Namely,

$$
\left\{\begin{array}{l}
\tilde{E}_{6}: f(x, y, z)=x^{3}+y^{3}+z^{3}+\lambda x y z \\
\tilde{E}_{7}: f(x, y, z)=x^{4}+y^{4}+z^{2}+\lambda x y z \\
\tilde{E}_{8}: f(x, y, z)=x^{6}+y^{3}+z^{2}+\lambda x y z
\end{array}\right.
$$

where $\lambda$ is a modular parameter.

Since a simply elliptic singularity has a semi-universal deformation, there exists the following commutative diagram which is called a Hamiltonian system is [SO]:

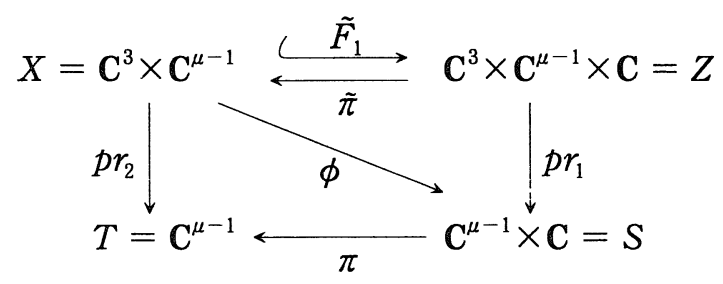

where

$$
\left\{\begin{array}{c}
\tilde{\pi}, \pi, p r_{1}, p r_{2}: \text { natural projections, } \\
\hat{F}_{1}\left(x, y, z, t_{1}, \ldots, t_{\mu-1}\right):=\left(x, y, z, t_{1}, \ldots, t_{\mu-1}, F_{1}\left(x, y, z, t_{1}, \ldots, t_{\mu-1}\right)\right) \\
F_{1}\left(x, y, z, t_{1}, \ldots, t_{\mu-1}\right):=f(x, y, z)+\sum_{j=1}^{\mu-1} t_{j} \phi_{j}
\end{array}\right.
$$

and

$$
\phi:=p r_{1} \circ \hat{F}_{1}: \text { semi-universal deformation of } f .
$$

Here $\mu=l+2$ and $\left\{\phi_{j}\right\}_{j=1}^{\mu}$ is a $\mathbf{C}$-basis of the Jacobi ring $\mathbf{C}[x, y, z] /\left(\frac{\partial f}{\partial x}, \frac{\partial f}{\partial y}, \frac{\partial f}{\partial z}\right)$ of $f$ such that $\operatorname{deg}\left(\phi_{j+1}\right) \leq \operatorname{deg}\left(\phi_{j}\right)$.

Let $C_{\phi}$ be the critical set of $\phi$ and $D_{\phi}$ the discriminant of $\phi$. The discriminant $D_{\phi}$ is a reduced irreducible hypersurface in $S$. Let $t^{\prime} \in T=$ $\mathbf{C}^{\mu-1}$ be a point which is not contained in the image of the ramification locus of $\left.\pi\right|_{D_{\phi}}$. Set

$$
L_{t^{\prime}}:=\left\{t^{\prime}\right\} \times \mathbf{C} \subset \mathbf{C}^{\mu-1} \times \mathbf{C}=S
$$

By choice of $t^{\prime}$, there are exactly $\mu$ intersection points of $L_{t^{\prime}}$ with the discriminant $D_{\phi}$. We denote these points by $p_{1}, \ldots, p_{\mu}$. The fibre $X_{p_{t}}=$ 
$\phi^{-1}\left(p_{i}\right)$ has a singularity which is an ordinary double point. Choose a generic point $p_{0} \in L_{t^{\prime}} \backslash\left\{p_{1}, \ldots, p_{\mu}\right\}$. Then the fibre $X_{p_{0}}=\phi^{-1}\left(p_{0}\right)$ is a 2-dimensional manifold and homotopically isomorphic to a bouquet of $\mu$ copies of sphere $S^{2}$. Hence, the only non-trivial homology group of $X_{p_{0}}$ is the group $H_{2}\left(X_{p_{0}}, \mathbb{Z}\right)$ which is a free $\mathbb{Z}$-module of rank $\mu$. The negative intersection numbers of cycles define a symmetric bilinear form $(\cdot, \cdot)$ on this module with signature $(l, 2,0)(\mu=l+2)$.

Next, we shall explain the relation between elliptic Dynkin diagrams and vanishing cycles. Choose a simple arc $l_{i}$ in $L_{t^{\prime}}$ form $p_{0}$ to $p_{i}$ not passing through the other $p_{j}$. Then

$$
X_{p_{0}} \subset \phi^{-1}\left(l_{i}\right) \rightarrow X_{p_{i}} \text { : contraction }
$$

induces the mapping

$$
c_{i}: H_{2}\left(X_{p_{0}}, \mathbb{Z}\right) \rightarrow H_{2}\left(X_{p_{i}}, \mathbb{Z}\right) .
$$

The kernel of this mapping is a $\mathbb{Z}$-submodule of $H_{2}\left(X_{p_{0}}, \mathbb{Z}\right)$ of rank 1 . Denote a generator of $\operatorname{Kernel}\left(c_{i}\right)$ by $e_{i}$, i.e.

$$
\operatorname{Kernel}\left(c_{i}\right)=\mathbb{Z}_{e_{i}}
$$

It can be shown that if $l_{1}, \ldots, l_{\mu}$ are chosen in such a way that $l_{i}$ and $l_{j}$ intersect only at $p_{0}$ for $i \neq j$, then $\left\{e_{1}, \ldots, e_{\mu}\right\}$ is a free $\mathbb{Z}$-basis of $H_{2}\left(X_{p_{0}}, \mathbb{Z}\right)$ and $H_{2}\left(X_{p_{0}}, \mathbb{Z}\right)=Q(R)$. By further specialization of this choice, the negative intersection matrix with respect to this basis yields an elliptic Dynkin diagram (see $[\mathrm{Eb}],[\mathrm{Ga}],[\mathrm{S}],[\mathrm{SO}],[\mathrm{Si}]$ ).

Now, we explain the classical Picard-Lefschetz formula. To each path $l_{i}$, we associate an element $\gamma_{i} \in \pi_{1}\left(L_{t^{\prime}}, p_{0}\right)$ by going along $l_{i}$ from $p_{0}$ to a point near $p_{i}$, then turning counterclockwise in a small circle around $p_{i}$ and then returning to $p_{0}$ along $l_{i}$. Then $\left\{\gamma_{1}, \ldots, \gamma_{\mu}\right\}$ is a set of generators of $\pi_{1}\left(S \backslash D_{\phi}, p_{0}\right)$. The mapping

$$
\phi: \phi^{-1}\left(S \backslash D_{\phi}\right) \rightarrow S \backslash D_{\phi}
$$

is the projection of a fibre bundle. Hence one gets a monodromy representation

$$
\rho: \pi_{1}\left(S \backslash D_{\phi}, p_{0}\right) \rightarrow \operatorname{Aut}\left(H_{2}\left(X_{p_{0}}, \underline{Z}\right)\right) .
$$

The map $\rho$ has the following explicit description: 
Theorem 3.1 (Classical Picard-Lefschetz formula $[\mathrm{P}]$ ).

$$
\begin{gathered}
\rho\left(\gamma_{i}\right)(\alpha)=\alpha-\left(\alpha, e_{i}\right) e_{i} \quad \text { for any } \alpha \in H_{2}\left(X_{p_{0}}, \mathbf{Z}\right) \\
(i=1, \ldots, \mu=l+2)
\end{gathered}
$$

Let us turn to a twisted version of the above formula following A. B. Givental [Gi]. Define $F: Z \rightarrow \mathbf{C}$ by

$$
F\left(x, y, z, t_{1}, \ldots, t_{\mu}\right):=F_{1}\left(x, y, z, t_{1}, \ldots, t_{\mu-1}\right)+t_{\mu}
$$

and

$$
\tilde{Z}:=Z \backslash F^{-1}(0)
$$

Since $\pi_{1}(\tilde{Z}) \cong \mathbf{Z}$, for a complex number $q \in \mathbf{C}^{*}$, we can define a representation

$$
\pi_{1}(\tilde{Z}) \rightarrow \operatorname{Aut}(\mathbf{C}): \quad 1 \mapsto q .
$$

This representation induces a local system $\mathcal{L}_{q}$ on $\tilde{Z}$. Define $\tilde{Z}^{r}=p r_{1}^{-1}$ $\left(S \backslash D_{\phi}\right) \cap \tilde{Z}$, then $p r_{1}: \tilde{Z}^{r} \rightarrow S \backslash D_{\phi}$ is a fibre bundle whose fibre is a 3-dimensional complex manifold. For simplicity, we also denote by $\mathcal{L}_{q}$ the restriction of $\mathcal{L}_{q}$ to the fibre $\tilde{Z}^{r}\left(p_{0}\right):=p r_{1}^{-1}\left(p_{0}\right)$. Then we get a monodromy representation

$$
p_{q}: \pi_{1}\left(S \backslash D_{\phi}\right) \rightarrow A u t_{\mathbf{Z}\left[q, q^{-1}\right]}\left(H_{3}\left(\tilde{Z}^{r}\left(p_{0}\right), \mathcal{L}_{q}\right)\right) .
$$

This monodromy representation can be regarded as a $q$-deformation of the classical one as follows.

Denote the restriction of the mapping $F$ to $\tilde{Z}^{r}\left(p_{0}\right)$ by

$$
\tilde{F}_{p_{0}}: \tilde{Z}^{r}\left(p_{0}\right) \rightarrow \mathbf{C}^{*} .
$$

By the choice of $p_{0}, \tilde{F}_{p_{0}}$ has exactly $\mu$ critical values. We denote these points by $p_{1}^{\prime}, \ldots, p_{\mu}^{\prime}$. Choose a simple arc $\gamma_{i}^{\prime}$ in $\mathbf{C}^{*}$ going from $p_{i}$ to a point near the origin, then turning counterclockwise in a small circle around it, and finally returning to $p_{i}^{\prime}$ along the same way, and define a cycle $\delta_{i} \in H_{3}\left(\tilde{Z}^{r}\left(p_{0}\right), \mathcal{L}_{q}\right)$ by carrying the vanishing cycle $e_{i}$ along $\gamma_{i}^{\prime}$. Then we obtain the following:

Theorem $3.2\left(\right.$ A. B. Givental [Gi]). (1) $H_{3}\left(\tilde{Z}^{r}\left(p_{0}\right), \mathcal{L}_{q}\right)=\oplus_{j=1}^{\mu} \mathbf{Z}\left[q, q^{-1}\right] \delta_{j}$

(2) Let $V$ be the upper triangular matrix with diagonal elements 1 and 
$\left(e_{i}, e_{j}\right)$ in the $(i, j)$-component for $i<j$. Define a $\mu \times \mu$-matrix $I_{q}=$ $q V+{ }^{t} V$. Then one has

$$
\rho_{q}\left(\gamma_{i}\right)\left(\delta_{j}\right)=\delta_{j}-\left(I_{q}\right)_{i, j} \delta_{i}
$$

$$
\left(\rho_{q}\left(\gamma_{i}\right)+q\right)\left(\rho_{q}\left(\gamma_{1}\right)-1\right)=0
$$

Givental's result in the above formulation is valid for all isolated 2-dimensional hypersurface singularities.

As an application in the simply elliptic case, we obtain by direct computations with the elliptic Dynkin diagrams the following proposition.

Proposition 3.1. Set

$$
g_{i}^{\prime}:=\rho_{q}\left(\gamma_{i}\right) \quad(i=1, \ldots, \mu=l+2)
$$

Then $g_{1}^{\prime}, \ldots, g_{\mu}^{\prime}$ satisfy the following relations:

(1) $\left(g_{i}^{\prime}+q\right)\left(g_{i}^{\prime}-1\right)=0$

$$
\begin{array}{ll}
\multicolumn{2}{c}{e_{i} \bigcirc} \\
e_{i} \bigcirc & \circ \\
e_{i} \bigcirc & \bigcirc
\end{array}
$$

(2) $\quad g_{i}^{\prime} g_{j}^{\prime}=g_{j}^{\prime} g_{i}^{\prime}$

$g_{i}^{\prime} g_{j}^{\prime} g_{i}^{\prime}=g_{j}^{\prime} g_{i}^{\prime} g_{j}^{\prime}$

(3) Let $t_{j}^{\prime}=g_{j}^{\prime} g_{j}^{\prime *}$, then $g_{i}^{\prime} t_{j}^{\prime} g_{i}^{\prime} t_{j}^{\prime}=t_{j}^{\prime} g_{i}^{\prime} t_{j}^{\prime} g_{i}^{\prime}$

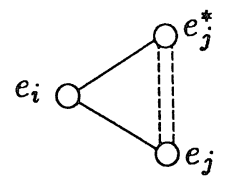

(4) Let $t_{j}^{\prime}=g_{i}^{\prime} t_{i}^{\prime} g_{i}^{\prime} t_{i}^{\prime-1}$, then $g_{k}^{\prime} t_{j}^{\prime}=t_{j}^{\prime} g_{k}^{\prime}$

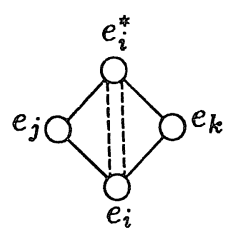

\section{§4. Elliptic Weyl Group and Elliptic Artin Group}

In this section, we will define elliptic Artin groups. Then we will explain the relation between elliptic Weyl groups and elliptic Artin groups.

Motivated by Proposition 3.1, we define the following group: 
Definition 4.1. Let $(R, G)$ be a 1-codimensional marked elliptic root system and $\Gamma(R, G)$ be its elliptic Dynkin diagram. Define an elliptic Artin group $A(R, G)$ by the following generators and their relations:

generators: $g_{\alpha}$

$$
\alpha \in \Gamma(R, G)
$$

relations: $\quad$ Set $t_{\alpha}:=g_{\alpha} g_{\alpha}^{*} \quad$ for $\alpha \in \Gamma\left(R_{a}\right)_{\max }$.

(E.1.0) $\quad g_{\alpha} g_{\beta}=g_{\beta} g_{\alpha}$

$\alpha \circ \circ^{\beta}$

(E.1.1) $\quad g_{\alpha} g_{\beta} g_{\alpha}=g_{\beta} g_{\alpha} g_{\beta}$

$\alpha \bigcirc$

(E.1.2) $\quad g_{\alpha} g_{\beta} g_{\alpha} g_{\beta}=g_{\beta} g_{\alpha} g_{\beta} g_{\alpha}$

$\alpha \overbrace{2 \pm 1} \rho \beta$

(E.1.3) $\quad g_{\alpha} g_{\beta} g_{\alpha} g_{\beta} g_{\alpha} g_{\beta}=g_{\beta} g_{\alpha} g_{\beta} g_{\alpha} g_{\beta} g_{\alpha}$

$\alpha \stackrel{\mathrm{Z}^{ \pm 1} \mathrm{O}}{ } \beta$

(E.2.1) $\quad g_{\beta} t_{\alpha} g_{\beta} t_{\alpha}=t_{\alpha} g_{\beta} t_{\alpha} g_{\beta}$

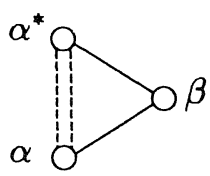

(E.2.2) $\quad g_{\beta} t_{\alpha} g_{\beta} g_{\alpha}=g_{\alpha} g_{\beta} t_{\alpha} g_{\beta}$

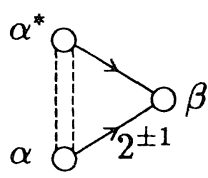

(E.2.3) $\quad g_{\beta} t_{\alpha} g_{\beta} t_{\alpha}=t_{\alpha} g_{\beta} t_{\alpha} g_{\beta}$ and $g_{\beta} t_{\alpha} g_{\beta} g_{\alpha}=g_{\alpha}^{*} g_{\beta} t_{\alpha} g_{\beta}$

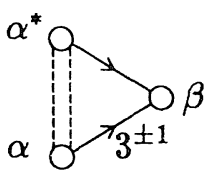

(E.2.4) $\quad g_{\beta} t_{\alpha} g_{\beta} t_{\alpha}=t_{\alpha} g_{\beta} t_{\alpha} g_{\beta}=g_{\alpha}^{*} g_{\beta} t_{\alpha} g_{\beta} g_{\alpha}$

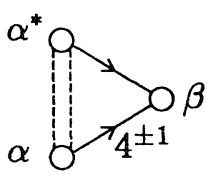

$$
\begin{aligned}
& g_{\alpha} t_{\gamma}=t_{\gamma} g_{\alpha} \text { and } g_{\gamma} t_{\alpha}=t_{\alpha} g_{\gamma} \\
& \text { where } t_{\gamma}=g_{\gamma} t_{\beta} g_{\gamma} t_{\beta}^{-1} \text { and } t_{\alpha}=g_{\alpha} t_{\beta} g_{\alpha} t_{\beta}^{-1} \\
& \text { for } t=1,2^{ \pm 1}, 3^{ \pm 1}
\end{aligned}
$$

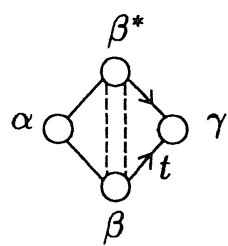

Here the relations (E.1.0) (E.1.3) are the same as (W.1) in Theorem 2.1 and the relations (E.2) (E.3) are new ones due to the broken bonds in the diagram $\Gamma(R, G)$.

Now we consider the relation between the elliptic Artin group $A(R$, 
$G)$ and the hyperbolic extension of elliptic Weyl group $\tilde{W}(R, G)$. Let $\hat{A}(R, G)$ be the quotient group of $A(R, G)$ by the following relation:

$$
g_{\alpha}^{2}=1 \quad \text { for } \alpha \in \Gamma(R, G)
$$

We denote by $\hat{g}_{\alpha}$ the element of $\hat{A}(R, G)$ corresponding to the element $g_{\alpha} \in A(R, G)$ for any $\alpha \in \Gamma(R, G)$. Then we get the following theorem:

Theorem 4.1. Let $(R, G)$ be a 1-codimensional marked elliptic root system. The correspondence $\hat{g}_{\alpha} \mapsto w_{\alpha}$ for $\alpha \in \Gamma(R, G)$ induces an isomorphism:

$$
\hat{A}(R, G) \cong \tilde{W}(R, G) .
$$

To prove this theorem, it is enough to show that the relations (E.0) $\sim(E .3)$ are equivalent to the elliptic relations (W.0) (W.3). We only show that the relations (E.2.1) (E.3) are equivalent to the relations (W.2.1) (W.3), since the equivalence for other relations is well known.

Lemma 4.1. Set $\hat{t}_{\alpha}:=\hat{g}_{\alpha} \hat{g}_{\alpha}{ }^{*}$ for $\alpha \in \Gamma\left(R_{a}\right)_{\max }$. Then we obtain the following:

$$
\begin{aligned}
& \left(\hat{g}_{\alpha} \hat{g}_{\beta} \hat{g}_{\alpha}^{*} \hat{g}_{\beta}\right)^{3}=1 \\
& \stackrel{g_{\beta}}{\Longleftrightarrow} \hat{t}_{\alpha} \hat{g}_{\beta} \hat{t}_{\alpha}=\hat{t}_{\alpha} \hat{g}_{\beta} \hat{t}_{\alpha} \hat{g}_{\beta} \\
& \left(\hat{g}_{\alpha} \hat{g}_{\beta} \hat{g}_{\alpha}^{*} \hat{g}_{\beta}\right)^{2}=1 \\
& \stackrel{g_{\alpha}}{\Longleftrightarrow} \hat{g}_{\beta} \hat{t}_{\alpha} \hat{g}_{\beta}=\hat{g}_{\beta} \hat{t}_{\alpha} \hat{g}_{\beta} \hat{g}_{\alpha}
\end{aligned}
$$

$$
\begin{aligned}
& \left(\hat{g}_{\alpha} \hat{g}_{\beta} \hat{g}_{\alpha}^{*} \hat{g}_{\beta}\right)^{3}=1 \text { and }\left(\hat{g}_{\alpha} \hat{g}_{\beta} \hat{g}_{\alpha}^{*} \hat{g}_{\beta} \hat{g}_{\alpha} \hat{g}_{\beta}\right)^{2}=1 \\
& \Longleftrightarrow \hat{t}_{\alpha} \hat{g}_{\beta} \hat{t}_{\alpha} \hat{g}_{\beta}=\hat{g}_{\beta} \hat{t}_{\alpha} \hat{g}_{\beta} \hat{t}_{\alpha} \text { and } \hat{g}_{\alpha}^{*} \hat{g}_{\beta} \hat{t}_{\alpha} \hat{g}_{\beta}=\hat{g}_{\beta} \hat{t}_{\alpha} \hat{g}_{\beta} \hat{g}_{\alpha}
\end{aligned}
$$

$$
\begin{aligned}
& \left(\hat{g}_{\alpha} \hat{g}_{\alpha}^{*} \hat{g}_{\beta}\right)^{2}=\left(\hat{g}_{\alpha}^{*} \hat{g}_{\beta} \hat{g}_{\alpha}\right)^{2}=\left(\hat{g}_{\beta} \hat{g}_{\alpha} \hat{g}_{\alpha}^{*}\right)^{2} \\
& \Longleftrightarrow \\
& \hat{t}_{\alpha} \hat{g}_{\beta} \hat{t}_{\alpha} \hat{g}_{\beta}=\hat{g}_{\beta} \hat{t}_{\alpha} \hat{g}_{\beta} \hat{t}_{\alpha}=\hat{g}_{\alpha}^{*} \hat{g}_{\beta} \hat{t}_{\alpha} \hat{g}_{\beta} \hat{g}_{\alpha}
\end{aligned}
$$

Proof.

Here we prove (1) and (2). The other cases follows from similar 
computations.

For simplicity, we set $a:=\hat{g}_{\alpha}, b:=\hat{g}_{\beta}$ and $t_{\alpha}:=\hat{g}_{\alpha} \hat{g}_{\alpha}^{*}$

Proof of (1)

$$
\begin{aligned}
\left(a b a^{*} b\right)^{3}=1 & \Leftrightarrow a b a^{*} b a b a^{*} b a b a^{*} b=1 \\
& \Leftrightarrow a b a^{*} a b a a^{*} b a a^{*} b a^{*}=1 \quad(\text { by }(E .1 .1)) \\
& \Leftrightarrow a b t_{a}^{-1} b t_{a} b t_{a} b a^{*}=1 \quad\left(\text { by the definition of } t_{a}\right) \\
& \Leftrightarrow b t_{a} b t_{a}=a a^{*} b a a^{*} b=t_{a} b t_{a} b
\end{aligned}
$$

Proof of (2)

$$
\begin{aligned}
a b t_{a} b=b t_{a} b a & \left.\Leftrightarrow a b a a^{*} b=b a a^{*} b a \quad \text { (by the definition of } t_{a}\right) \\
& \Leftrightarrow a b a b b a^{*} b=b a a^{*} b a \quad(\text { by }(E .0)) \\
& \Leftrightarrow b a b a b a^{*} b=b a a^{*} b a \quad(\text { by }(E .1 .2)) \\
& \Leftrightarrow b a b a^{*} b=a^{*} b a \\
& \Leftrightarrow\left(a b a^{*} b\right)^{2}=1 \quad(\text { by }(E .0)) .
\end{aligned}
$$

Lemma 4.2. Set $\hat{t}_{\beta}:=\hat{g}_{\beta} \hat{g}_{\beta}^{*}$ for $\beta \in \Gamma\left(R_{a}\right)_{\max }$. Then we obtain the following:

$$
\begin{aligned}
& \left(\hat{g}_{\alpha} \hat{g}_{\beta} \hat{g}_{\alpha} \hat{g}_{\beta}^{*} \hat{g}_{\gamma} \hat{g}_{\beta}^{*}\right)^{2}=1 \text { and }\left(\hat{g}_{\alpha} \hat{g}_{\beta}^{*} \hat{g}_{\alpha} \hat{g}_{\beta} \hat{g}_{\gamma} \hat{g}_{\beta}^{*}\right)^{2}=1 \\
& \stackrel{\Longleftrightarrow}{\hat{g}_{\alpha} \hat{t}_{\gamma}=\hat{t}_{\gamma} \hat{g}_{\alpha} \text { and } \hat{g}_{\gamma} \hat{t}_{\alpha}=\hat{t}_{\alpha} \hat{g}_{\gamma}}
\end{aligned}
$$

where $\hat{t}_{\alpha}=\hat{g}_{\alpha} \hat{t}_{\beta} \hat{g}_{\alpha} \hat{t}_{\beta}^{-1}$ and $\hat{t}_{\gamma}=\hat{g}_{\gamma} \hat{t}_{\beta} \hat{g}_{\gamma} \hat{t}_{\beta}^{-1}$ for $t=1,2^{ \pm 1}, 3^{ \pm 1}$

Proof.

For simplicity, we set $a:=\hat{g}_{\alpha}, b:=\hat{g}_{\beta}, c:=\hat{g}_{r}, t_{b}:=\hat{g}_{\beta} \hat{g}_{\beta}^{*}, t_{a}:=$ $a t_{b} a t_{b}^{-1}$ and $t_{c}:=c t_{b} c t_{b}^{-1}$.

$$
\begin{aligned}
a t_{c}=t_{c} a & \Leftrightarrow a c b b^{*} c b^{*} b=c b b^{*} c b^{*} b a \text { (by the definition of } t_{c} \text { ) } \\
& \Leftrightarrow c a b b^{*} c b^{*} b=c b b^{*} c b^{*} b a \quad(\text { by }(E .1 .0)) \\
& \Leftrightarrow a b b^{*} c b^{*} b=b b^{*} c b^{*} b a \\
& \Leftrightarrow b a b b^{*} c b^{*}=b^{*} c b^{*} b a b \\
& \Leftrightarrow a b a b^{*} c b^{*}=b^{*} c b^{*} a b a \quad(\text { by }(E .1 .1)) \\
& \Leftrightarrow\left(a b a b^{*} c b^{*}\right)^{2}=1
\end{aligned}
$$

We can prove that the relation $c t_{a}=t_{a} c$ is equivalent to the relation $\left(a b^{*} a b c b\right)^{2}=1$ in the same way.

From Lemma 4.1 and Lemma 4.2, we obtain Theorem 4.1. 


\section{§5. Extended Artin Group and Elliptic Artin Group}

In this section, we will compare the elliptic Artin group $A(R, G)$ with the extended Artin group $A\left(R_{a}\right)$. To this purpose, first we define the extended Artin group $A\left(R_{a}\right)$ following $\mathrm{H}$. van der Lek [L].

Definition 5.1. Let $R_{a}$ be a affine root system and $Q\left(R_{a}\right)$ be its root lattice. Set $V_{\mathrm{C}}:=Q\left(R_{a}\right) \otimes \mathrm{C}$. The affine Weyl group $W\left(R_{a}\right)$ and its extension $W\left(R_{a}\right) \ltimes Q\left(R_{a}\right)$ act on $V_{\mathrm{c}}$ Then we define the extended Artin group by

$$
A\left(R_{a}\right):=\pi_{1}\left(V_{\mathrm{c}}^{r} / W\left(R_{a}\right) \ltimes Q\left(R_{a}\right)\right)
$$

where $V_{\mathrm{c}}^{r}$ is the set of regular elements of $V_{\mathrm{C}}$.

Remark 5.1. Note that this is the fundamental group of the complement of the discriminant in the semi-universal deformation of a simply elliptic singularity ([Lo], cf. also $[\mathrm{S} 0],[\mathrm{S} 1],[\mathrm{S} 2]$ ).

Let us recall the following result: Let $C:=\left(c_{i, j}\right)_{0 \leq i, j \leq l}$ be an affine Cartan matrix and $M:=\left(m_{i, j}\right)_{0 \leq i, j \leq l}$ be the Coxeter matrix determined by $c_{i, j}$ as follows:

$$
m_{i, j}=2,3,4,6, \infty \quad \text { if } \quad c_{i, j} c_{j, i}=0,1,2,3, \geq 4, \quad \text { respectively } .
$$

Theorem 5.1 ( $H_{\text {. }}$ van der Lek $[\mathbf{L}]$ ). Let $R_{a}$ be an affine root system and $M\left(R_{a}\right)$ the corresponding Coxeter matrix. The extended Artin group $A\left(R_{a}\right)$ associated with $R_{a}$ is generated by $\left\{S_{0}, S_{1}, \ldots, S_{l}, T_{0}, T_{1}, \ldots, T_{1}\right\}$ which satisfy the following relations:

(A.1) $\quad S_{i} S_{j} S_{i} \cdots S_{j} S_{i} S_{j} \cdots \quad$ each side $m_{i, j}$ factors if $i \neq j$

(A.2) $T_{i} T_{j}=T_{j} T_{i}$

(A.3) $\quad S_{i} T_{j} T_{i}^{r}=T_{j} T_{i}^{r} S_{i} \quad c_{j, i}=-2 r$

(A.4) $S_{i} T_{j} T_{i}^{r} S_{i}=T_{j} T_{i}^{r+1} \quad c_{j, i}=-(2 r+1)$

Let us consider again an elliptic root $\operatorname{system}(R, G)$ of 1-codimension.

We define $t_{\alpha} \in A(R, G)$ for all $\alpha \in \Gamma\left(R_{a}\right)$ as follows: Assume $\alpha_{0} \in$ $\Gamma\left(R_{a}\right)_{\max }, t_{\alpha_{0}}$ has already been defined by

$$
t_{\alpha_{0}}:=g_{\alpha_{0}} g_{\alpha_{0}}^{*}
$$

Suppose the vertices $\left\{\alpha_{0}, \ldots, \alpha_{k}\right\} \subset \Gamma\left(R_{a}\right) \backslash \Gamma\left(R_{a}\right)_{\max }$ are arranged in the 
following way:

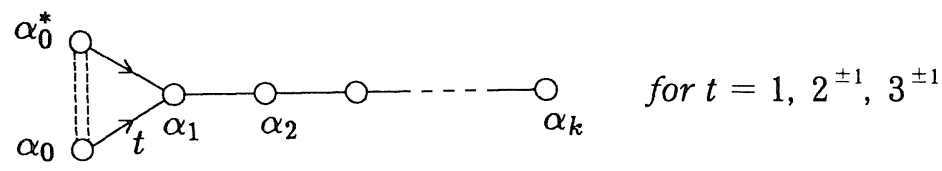

Then we set

$$
t_{\alpha_{j+1}}:=g_{\alpha_{j+1}} t_{\alpha_{j}} g_{\alpha_{j+1}} t_{\alpha_{j}}^{-1}
$$

inductively. The following lemma is the key step in the proof of our Theorem 5.2.

Lemma 5.1. ( $i)$ Let $N(R, G)$ be the subgroup of $A(R, G)$ generated by $\left\{t_{\alpha} \mid \alpha \in \Gamma(R, G)\right\}$. Then $N(R, G)$ is a free abelian subgroup isomorphic to the lattice $Q\left((R, G)_{a}\right)$.

(ii) Let $\beta \in \Gamma\left((R, G)_{a}\right)_{\max }$ Then one has the following formulae for $\alpha \in \Gamma\left((R, G)_{a}\right) \backslash \Gamma\left((R, G)_{a}\right)_{\max }$ :

$$
\stackrel{\circ}{\circ} \stackrel{\circ}{\beta} \quad g_{\beta} t_{\alpha}=t_{\alpha} g_{\beta}
$$

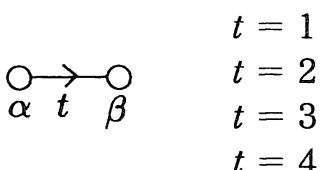

$$
\begin{aligned}
& t=4
\end{aligned}
$$$$
g_{\beta} t_{\alpha} g_{\beta}=t_{\alpha} t_{\beta}
$$$$
g_{\beta} t_{\alpha} t_{\beta}=t_{\alpha} t_{\beta} g_{\beta}
$$$$
g_{\beta} t_{\alpha} t_{\beta} g_{\beta}=t_{\alpha} t_{\beta}^{2}
$$$$
g_{\beta} t_{\alpha} t_{\beta}^{2}=t_{\alpha} t_{\beta}^{2} g_{\beta}
$$

$$
\underset{\alpha}{\longrightarrow} \rightarrow \mathrm{O}_{\beta} \quad t=1,2,3,4 \quad g_{\alpha} t_{\beta} g_{\alpha}=t_{\alpha} t_{\beta}
$$

(iii) Set $c(R, G)=\prod_{\alpha \in \Gamma(R, G) \backslash \Gamma\left(R_{\alpha}\right)_{\max }} g_{\alpha} \prod_{\alpha \in \Gamma\left(R_{a}\right)_{\max }} g_{\alpha} g_{\alpha}$, then its power $c(R$, $G)^{m(R, G)}$ is an element of the center of $A(R, G)$ and belongs to $N(R, G)$. In particular, $c(R, G)^{m(R, G)}$ is expressed as

$$
c(R, G)^{m(R, G)}=\prod_{\alpha \in \Gamma\left(R_{\alpha}\right)} t_{\alpha}^{n_{\alpha}}
$$

where the $n_{\alpha}$ are the coefficients of the generator $b$ of the imaginary roots in $(R, G)_{a}$. 
Proof.

Proof of (i)

Here we prove $(i)$ in the case of $t=1$. We can prove the other cases similarly to the case of $t=1$.

For simplicity, we set $a_{i}:=g_{\alpha_{i}}$ and $t_{i}:=t_{\alpha_{i}}, i=0,1, \ldots, k$.

First, we prove $t_{0}$ and $t_{1}$ are commuting.

$$
\begin{aligned}
t_{1} t_{0} & \left.=a_{1} t_{0} a_{1} t_{0}^{-1} t_{0} \quad \text { (by the definition of } t_{1}\right) \\
& =a_{1} t_{0} a_{1} \\
& =t_{0} a_{1} t_{0} a_{1} t_{0}^{-1} \quad(\text { by }(E .2 .1)) \\
& =t_{0} t_{1} \quad\left(\text { by the definition of } t_{1}\right) .
\end{aligned}
$$

Next, we prove that $a_{2}$ and $t_{1}$ satisfy the following relation:

$$
a_{2} t_{1} a_{2} t_{1}=t_{1} a_{2} t_{1} a_{2}
$$

By (E.1.1), (E.2.1) and commutativity of $t_{0}$ and $t_{1}$, one obtains the following:

$$
\begin{aligned}
& a_{2} t_{1} a_{2} t_{1}-t_{1} a_{2} t_{1} a_{2} \\
= & a_{2} a_{1} t_{0} a_{1} t_{0}^{-1} a_{2} t_{1}-a_{1} t_{0} a_{1} t_{0}^{-1} a_{2} t_{1} a_{2} \\
= & a_{2} a_{1} t_{0} a_{1} a_{2} t_{1} t_{0}^{-1}-a_{1} t_{0} a_{1} a_{2} t_{1} a_{2} t_{0}^{-1} \\
= & \left(a_{2} a_{1} t_{0} a_{1} a_{2} t_{1}-a_{1} t_{0} a_{1} a_{2} t_{1} a_{2}\right) t_{0}^{-1} \\
= & \left(a_{2} a_{1} t_{0} a_{1} a_{2} a_{1} t_{0} a_{1} t_{0}^{-1}-a_{1} t_{0} a_{1} a_{2} a_{1} t_{0} a_{1} t_{0}^{-1} a_{2}\right) t_{0}^{-1} \\
= & \left(a_{2} a_{1} t_{0} a_{1} a_{2} a_{1} t_{0} a_{1}-a_{1} t_{0} a_{1} a_{2} a_{1} t_{0} a_{1} a_{2}\right) t_{0}^{-2} \\
= & \left(a_{2} a_{1} t_{0} a_{2} a_{1} a_{2} t_{0} a_{1}-a_{1} t_{0} a_{2} a_{1} a_{2} t_{0} a_{1} a_{2}\right) t_{0}^{-2} \\
= & \left(a_{2} a_{1} a_{2} t_{0} a_{1} t_{0} a_{2} a_{1}-a_{1} a_{2} t_{0} a_{1} t_{0} a_{2} a_{1} a_{2}\right) t_{0}^{-2} \\
= & \left(a_{1} a_{2} a_{1} t_{0} a_{1} t_{0} a_{2} a_{1}-a_{1} a_{2} t_{0} a_{1} t_{0} a_{1} a_{2} a_{1}\right) t_{0}^{-2} \\
= & a_{1} a_{2}\left(a_{1} t_{0} a_{1} t_{0}-t_{0} a_{1} t_{0} a_{1}\right) a_{2} a_{1} t_{0}^{-2} \\
= & a_{1} a_{2}\left(t_{0} a_{1} t_{0} a_{1}-t_{0} a_{1} t_{0} a_{1}\right) a_{2} a_{1} t_{0}^{-2} \\
= & 0 .
\end{aligned}
$$

Hence, we have

$$
a_{2} t_{1} a_{2} t_{1}=t_{1} a_{2} t_{1} a_{2}
$$

From this relation and the definition of $t_{2}$, one has

$$
t_{2} t_{1}=a_{2} t_{1} a_{2}=t_{1} a_{2} t_{1} a_{2} t_{1}^{-1}=t_{1} t_{2}
$$

Hence we have 


$$
t_{1} t_{2}=t_{2} t_{1}
$$

From similar computations, we obtain

$$
a_{i+1} t_{i} a_{i+1} t_{i}=t_{\imath} a_{i+1} t_{i} a_{i} a_{i+1} \quad \text { for } i=2, \ldots, k \text {. }
$$

By this relations and (E.1.0), we obtain the commutativity of $t_{i}$ and $t_{j}$ for $i, j=0,1, \ldots, k$. Freeness of $N(R, G)$ follows from the Theorem 2.1 ( $i)$ and Theorem 4.1 .

\section{Proof of (ii)}

(0) and (2) are trivial from (E.1.0) and the definition of $t_{\alpha}$. Here we only prove the case of $t=2$ in (1). For simplicity, we set $a:=g_{\alpha}, b:=g_{\beta}$ and $t_{a}:=t_{\alpha}$.

Since $\beta \in \Gamma\left(R_{a}\right)_{\max }$ and $\alpha \in \Gamma\left(R_{a}\right) \backslash \Gamma\left(R_{a}\right)_{\max }$, we have the following:

$$
\begin{aligned}
b t_{a} t_{b} & =b a t_{b} a t_{b}^{-1} t_{b} \\
& =b a t_{b} a \\
& =a t_{b} a b(\text { by }(E .2 .2)) \\
& =t_{a} t_{b} b .
\end{aligned}
$$

Hence we have

$$
b t_{a} t_{b}=t_{a} t_{b} b
$$

Furthermore, by the definition of $t_{a}$, we have

$$
a t_{b} a=t_{a} t_{b}
$$

\section{Proof of (iii)}

This part will only be relevant in Section 6 . Here we only prove the case of $G_{2}^{(1,3)}$ (see Table in appendix). The other cases follows from similar computations.

In the case of $G_{2}^{(1,3)}$, the set of exponents is $\left(m_{0}, m_{1}, m_{2}\right)=(1,2,3)$ and the Coxeter element is

$$
c=a_{0} a_{1} t_{2}
$$

By the relations of (E.1.1) and (ii), (0) and (2) in this lemma, we have

$$
\begin{aligned}
c^{3} & =a_{0} a_{1} t_{2} a_{0} a_{1} t_{2} a_{0} a_{1} t_{2} \\
& =a_{0} a_{1} a_{0} t_{2} a_{1} t_{2} a_{0} a_{1} t_{2}
\end{aligned}
$$




$$
\begin{aligned}
& =a_{1} a_{0} a_{1} t_{2} a_{1} t_{2} a_{0} a_{1} t_{2} \\
& =a_{1} a_{0} t_{1} t_{2} t_{2} a_{0} a_{1} t_{2} \\
& =a_{1} a_{0} t_{1} a_{0} t_{2} t_{2} a_{0} a_{1} t_{2} \\
& =a_{1} t_{0} t_{1} t_{2} t_{2} g_{1} t_{2} \\
& =a_{1} t_{0} a_{1} a_{1}^{-1} t_{1} t_{2} t_{2} g_{1} t_{2} \\
& =t_{0} t_{1} a_{1}^{-1} t_{1} t_{2} t_{2} g_{1} t_{2} \\
& =t_{0} t_{1} t_{2} a_{1} t_{2} g_{1} t_{2} \\
& =t_{0} t_{1} t_{2} t_{1} t_{2} t_{2} .
\end{aligned}
$$

By the commutativity of $t_{i}$ and $t_{j}$, we obtain

$$
c^{3}=t_{0} t_{1}^{2} t_{2}^{3}
$$

By (ii) (1), we can prove that $c(R, G)^{m(R, G)}$ is central in $A(R, G)$.

Combining H. van der Lek's Theorem 5.1 and Lemma 5.2, we obtain the following surjective homomorphism:

$$
\begin{aligned}
\phi: A\left((R, G)_{a}\right) & \rightarrow A(R, G) \\
S_{\alpha} & \mapsto g_{\alpha} \\
T_{\alpha} & \mapsto t_{\alpha}
\end{aligned}
$$

for any $\alpha \in \Gamma\left((R, G)_{a}\right)$.

To construct the inverse homomorphism of $\phi$, let us define the element $S_{\alpha}^{*}$ of the extended Artin group $A\left((R, G)_{a}\right)$ by

$$
S_{\alpha}^{*}:=S_{\alpha}^{-1} T_{\alpha}
$$

for $\alpha \in \Gamma\left((R, G)_{a}\right)_{\max }$.

Now we obtain the following lemma:

Lemma 5.2. $S_{\alpha}, T_{\alpha}, S_{\beta}^{*}, S_{\beta}$, and $T_{\beta}$ for $\alpha \in \Gamma\left((R, G)_{a}\right), \quad \beta \in$ $\Gamma\left((R, G)_{a}\right)_{\max }$ satisfy the relations $(E .1 .0) \sim(E .3)$ in the Definition 4.1 of the elliptic Artin $A(R, G)$.

\section{Proof.}

Here we prove that $S_{\beta}^{*}$ satisfies the relations (E.1.1), (E.1.2), (E.2.1), (E.2.2) and (E.3). The other cases follows from similar computations.

For simplicity, we set $a:=S_{\alpha}, T_{a}:=T_{\alpha}, b:=S_{\beta}, b^{*}:=S_{\beta}^{*}$ and $T_{b}:=T_{\beta}$ for $\alpha \in \Gamma\left((R, G)_{a}\right) \backslash \Gamma\left((R, G)_{a}\right)_{\max }$. and $\beta \in \Gamma\left((R, G)_{a}\right)_{\max }$.

Proof of (E.1.1.)

In this case, $\left(\alpha^{\vee}, \beta\right)=\left(\alpha, \beta^{\vee}\right)=-1$. By(A.1), (A.2) and (A.4), $a, b$, 
$b^{*}, T_{a}$, and $T_{b}$ satisfy the following relations:

$$
a b a=b a b, T_{a} T_{b}=T_{b} T_{a}, a T_{b} a=b t_{a} b=T_{a} T_{b} .
$$

By these relations, we will show that $a$ and $b^{*}$ satisfy the following relation:

$$
a b^{*} a=b^{*} a b^{*}
$$

Now, we have

$$
\begin{aligned}
b T_{a} b & =b a T_{b} a T_{b}^{-1} b \\
& =b a b b^{*} a b^{*-1} b^{-1} b \\
& =a b a b^{*} a b^{*-1}
\end{aligned}
$$

On the other hand, we have

$$
\begin{aligned}
b T_{a} b & =T_{a} T_{b} \\
& =a T_{b} a T_{b}^{-1} T_{b} \\
& =a b b^{*} a .
\end{aligned}
$$

Hence we have

$$
a b a b^{*} a b^{*-1}=a b b^{*} a .
$$

Therefore, we obtain

$$
a b^{*} a=b^{*} a b^{*}
$$

Proof of (E.1.2)

In this case, $\left(\alpha^{\vee}, \beta\right)=-1$ and $\left(\alpha, \beta^{\vee}\right)=-2$. By (A.1) (A.4), $a$, $b, b^{*}, T_{a}$, and $T_{b}$ satisfy the following relations:

$$
b T_{a} T_{b}=T_{a} T_{b} b, a T_{b} a=T_{a} T_{b}, T_{a} T_{b}=T_{b} T_{a}, a b a b=b a b a .
$$

By these relations, we will show that $a$ and $b^{*}$ satisfy the following relation:

$$
a b^{*} a b^{*}=b^{*} a b^{*} a .
$$

We have 


$$
b T_{a} T_{b}=b a T_{b} a=b a b b^{*} a
$$

Moreover, we have

$$
T_{b} T_{a} b=b b^{*} a b b^{*} a b^{*-1} b^{-1} b=b b^{*} a b b^{*} a b^{*-1}
$$

Since $b T_{a} T_{b}=T_{b} T_{a} b$, we obtain

$$
a b b^{*} a b^{*}=b^{*} a b b^{*} a
$$

Hence we have the following relation:

$$
a b a^{-1} a b^{*} a b^{*}=b^{*} a b b^{*} a .
$$

On the other hand, we have

$$
b a b b^{*} a=b T_{a} T_{b}=T_{a} T_{b} b=a T_{b} a b=a b b^{*} a b .
$$

Since $b a b a=a b a b$, we have

$$
\begin{gathered}
b a b b^{*} a=a b b^{*} a b \\
\Longleftrightarrow \\
a b a b a^{-1} b^{*} a=a b b^{*} a b \\
\Longleftrightarrow \\
a b a^{-1} b^{*} a=b^{*} a b .
\end{gathered}
$$

Hence we have

$$
a b a^{-1}=b^{*} a b a^{-1} b^{*-1}
$$

By (1) and (2), we have

$$
\begin{gathered}
b^{*} a b a^{-1} b^{*-1} a b^{*} a b^{*}=b^{*} a b b^{*} a \\
\Longleftrightarrow \\
a^{-1} b^{*-1} a b^{*} a b^{*}=b^{*} a .
\end{gathered}
$$

Therefore we obtain

$$
a b^{*} a b^{*}=b^{*} a b^{*} a
$$

Proof of (E.2.1)

In this case, $\left(\alpha^{\vee}, \beta\right)=\left(\alpha, \beta^{\vee}\right)=-1$. By (A.4), $a$ and $T_{b}$ satisfy the 
following relation:

$$
a T_{b} a=T_{a} T_{b}
$$

By commutativity of $T_{a}$ and $T_{b}$, we have

$$
a T_{b} a T_{b}=T_{a} T_{b} T_{b}=T_{b} T_{a} T_{b}=T_{b} a T_{b} a T_{b}^{-1} T_{b}=T_{b} a T_{b} a .
$$

Hence we obtain

$$
a T_{b} a T_{b}=T_{b} a T_{b} a
$$

Proof of (E.2.2)

In this case, $\left(\alpha^{\vee}, \beta\right)=-1$ and $\left(\alpha, \beta^{\vee}\right)=-2$. By (A.3) and (A.4), $a$, $b, T_{a}$ and $T_{b}$ satisfy the following relations:

$$
a T_{b} a=T_{a} T_{b}, b T_{a} T_{b}=T_{a} T_{b} b .
$$

By these relations, we have

$$
a T_{b} a b=T_{a} T_{b} b=b T_{a} T_{b}=b a T_{b} a T_{b}^{-1} T_{b}=b a T_{b} a .
$$

Therefore, we obtain

$$
a T_{b} a b=b a T_{b} a
$$

Proof of (E.3)

In this case, $\left(\alpha^{\vee}, \gamma\right)=\left(\alpha, \gamma^{\vee}\right)=0$. By (A.3), we have $a T_{c}=T_{c} a$ and $c T_{a}=T_{a} c$ where $c=S_{\gamma}$ and $T_{c}=T_{r}$

Combining Lemma 5.1 and Lemma 5.2, we obtain the following theorem:

Theorem 5.2. Let $(R, G)$ be a 1-codimensional marked elliptic root system and $(R, G)_{a}$ the corresponding affine root system. Then the group $A(R, G)$ is isomorphic to the extended Artin group $A\left((R, G)_{a}\right)$.

Therefore, we obtain generators and their relations of an extended Artin group in terms of the corresponding elliptic Dynkin diagram.

Remark 5.2. In $[\mathrm{L}], \mathrm{H}$. van der Lek studies a more general case, where $W\left(R_{a}\right)$ is replaced by a Weyl group of an arbitrary Kac-Moody algebra. To such a group, one can attach an affinization of the 
Kac-Moody Dynkin diagram [S12] as an analogue of the elliptic Dynkin diagram. Our main result, Theorem 5.2, should go through in that situation as well.

\section{§6. Elliptic Hecke Algebra}

In [C1], [C2], [C3] I. Cherednik introduced double affine Hecke algebras and proved Macdonald's inner product conjecture. In this section, we shall define the elliptic Hecke algebra which can be proved to be a subalgebra of his algebra.

Definition 6.1. Let $(R, G)$ be a 1-codimensional marked elliptic root system. For $q \in \mathbb{C}^{*}$, the elliptic Hecke algebra $H_{q}(R, G)$ associated with $(R, G)$ is the quotient of the group algebra $\mathbb{C}[A(R, G)]$ by the relations

$$
\left(g_{\alpha}+q\right)\left(g_{\alpha}-1\right)=0 \quad \text { for } \alpha \in \Gamma(R, G) .
$$

Remark 6.1. (1) One may treat $q$ as an element which is purely transcendental over $\mathbb{C}$. In this case, the above elliptic Hecke algebras are defined as algebras over $\mathbf{C}(q)$.

(2) An elliptic Hecke algebra may be regarded as a subalgebra of Cherednik's double affine Hecke algebra. A double affine Hecke algebra contains two parameters. Two parameters in an elliptic Hecke algebra appear from the local system $\mathcal{L}_{q}$ and the $m(R, G)$-th power of the Coxeter element $c(R, G)$.

Let $C(R, G):=\left(c_{i, j}\right)$ be the Cartan matrix corresponding to an elliptic Dynkin diagram $\Gamma(R, G)$ for a fixed ordering of vertices of $\Gamma(R, G)$ and $T$ be an upper triangular matrix with diagonal elements 1 and the $(i, j)$-component $=c_{i, j}$ for $i<j$. Define $\mu \times \mu$-matrix

$$
C_{q}(R, G):=q \cdot T+(C(R, G)-T),
$$

where $\mu=$ the number of vertices of $\Gamma(R, G)$.

On the vector space

$$
V(R, G):=\oplus_{\alpha \in \Gamma(R, G)} \mathbf{C} \alpha,
$$

for any $\alpha \in \Gamma(R, G)$, define the element $A_{\alpha}$ of $\operatorname{Aut}(V(R, G))$ as follows: for any $\beta \in \Gamma(R, G)$, 


$$
A_{\alpha}(\beta):=\beta-C_{q}(R, G)_{\alpha, \beta} \cdot \alpha
$$

where $C_{q}(R, G)_{\alpha, \beta}$ is the $(\alpha, \beta)$-component of $C_{q}(R, G)$. Then we obtain the following proposition by direct calculations:

Proposition 6.1. Let $(R, G)$ be a 1-codimensional marked elliptic root system. Then one has

(1)

$$
\begin{gathered}
\rho_{q}: A(R, G) \longrightarrow \operatorname{Aut}(V(R, G)) \\
\rho_{q}\left(g_{\alpha}\right)=A_{\alpha}
\end{gathered}
$$

is a finite dimensional irreducible representation of $A(R, G)$ over $\mathbf{C}$.

(2) The above representation induces the following commutative diagram:

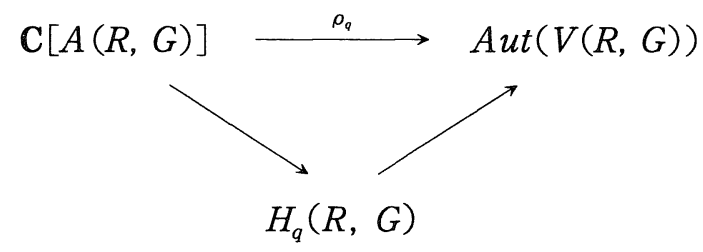

In particular, one obtains a finite dimensional irreducible representation of $H_{q}(R, G)$.

Table 1-codimensional elliptic Dynkin diagrams

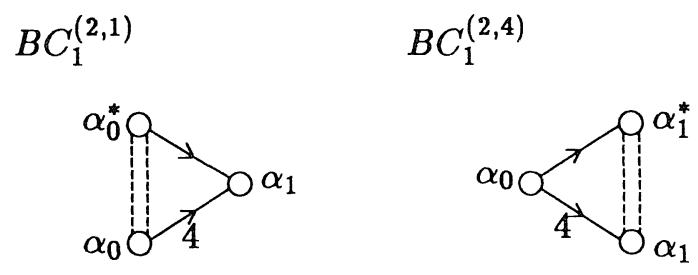

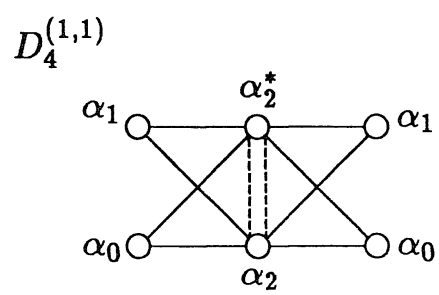



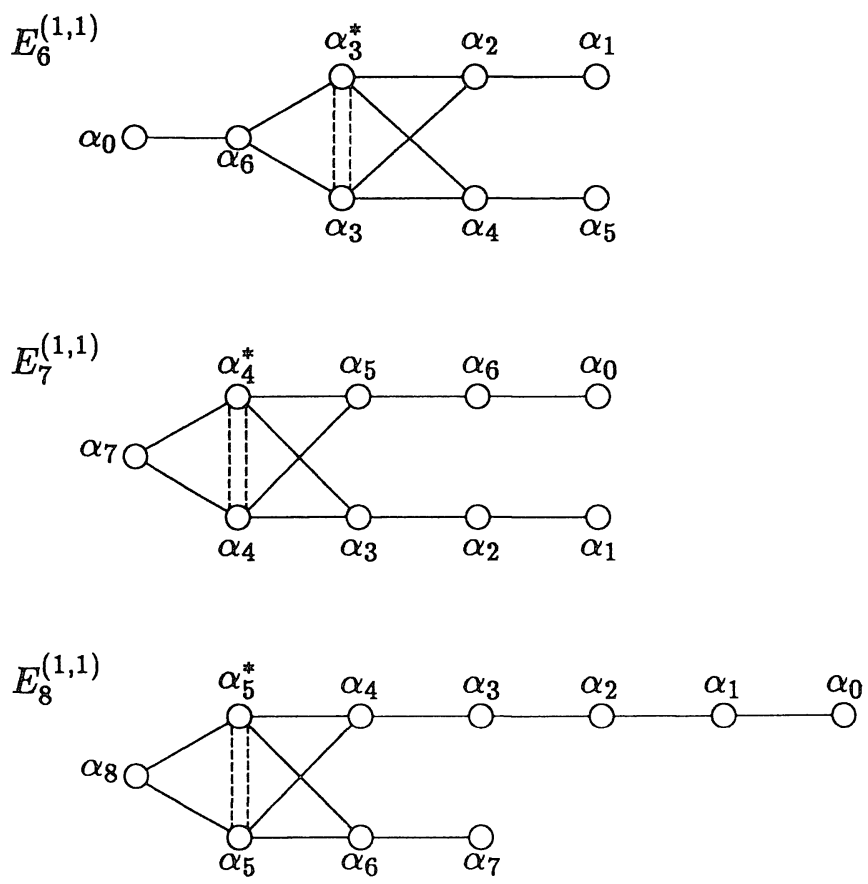

$F_{4}^{(1,1)}$

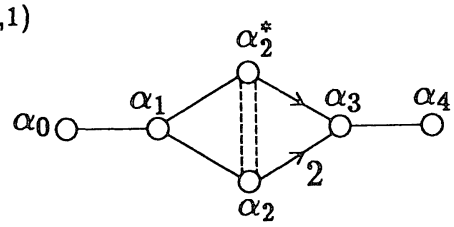

$\mathbb{F}_{4}^{(1,2)}$

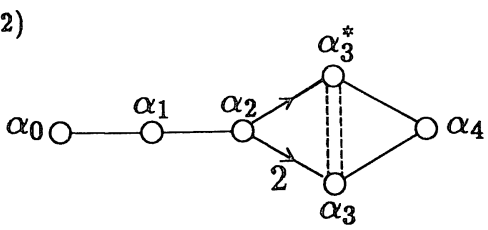

$G_{2}^{(1,1)}$

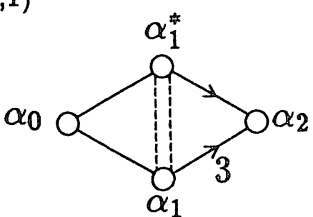

$F_{4}^{(2,2)}$

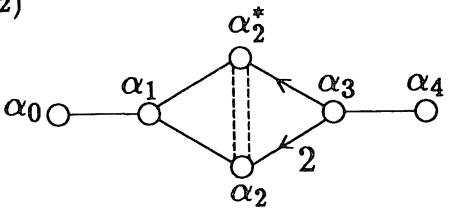

$F_{4}^{(2,1)}$

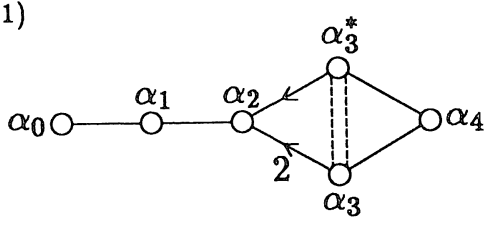

$G_{2}^{(1,3)}$

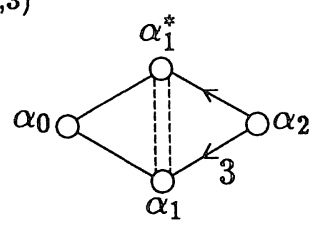



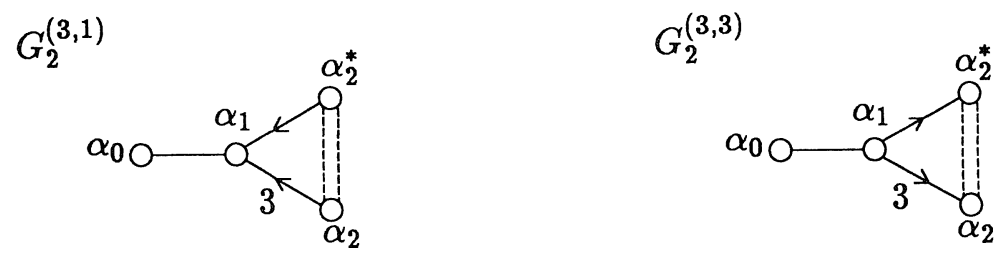

\section{References}

[BM] Berman, S. and Moody, R. V., Lie algebras graded by finite root systems and intersection matrix algebras of Slodowy, Invent. Math., 108 (1992), 323-347.

[Bor1] Borcherds, R., Vertex algebras, Kac-Moody algebras and the Monster, Proc. Nat. Acad. Sci. USA, 83 (1986), 3068-3071.

[Bor2] - Monstrous moonshine and monstrous Lie superalgebras, Invent. Math., 109 (1992), 405-444.

[C1] Cherednik, I., Double Affine Hecke Algebras, Knizhnik-Zamolodchikov Equations, and Macdonald's Operators, Internat. Math. Res. Notice, No. 9 (1992), 171-179.

[C2] - Double Affine Hecke Algebras and Macdonald's conjecture, Ann. Math., 141 (1995), 191-216.

[C3] - Macdonald's evaluation conjectures and difference Fourier transform, Invent. Math., 122 (1995), 119-145.

[Eb] Ebeling, W., The Monodromy Groups of Isolated Singularities of Complete Intersections, Lecture Notes in Math., 1293 (1987).

[Ga] Gabrielov, A. M., Dynkin diagrams unimodal singularities, Funct. Anal. Appl., 8 (1974), 192-196.

[Gi] Givental, A. B., Twisted Picard-Lefschetz formulas, Funct Anal. Appl., 22 (1987), 10-18.

[Lo] Looijenga, E. J. On the semi-universal deformation of a simple elliptic singularity II, Topology, 17 (1978), 23-40.

[MEY] Moody, R. V., Eswara Rao, S. and Yokonuma, T., Toroidal Lie algebras and vertex representation, Geom. Dedicata., 35 (1990), 283-307.

[P] Pham, F., Formules de Picard-Lefschetz généralisées et ramification de intégrales, Bull. Soc. Math. France, 93 (1965), 333-367.

[S] Saito, K., Einfach Elliptische Singularitaten, Invent. Math., 23 (1974), 289-325

[S0] - Period Mapping Associated to a Primitive Form, Publ. RIMS, Kyoto Univ., 19 (1983), 1231-1264.

[S1] - Extended Affine Root Systems I, Publ. RIMS, Kyoto Univ., 21 (1985), 75-179. 
[S2] Saito, K., Extended Affine Root Systems II (Flat Invariants), Publ. RIMS, Kyoto Univ., 26 (1990), 15-78.

[ST] Saito, K. and Takebayashi, T., Extended Affine Root Systems III (Elliptic Weyl Groups), Publ. RIMS, Kyoto Univ., 33 (1997), 301-329.

[SY] Saito, K. and Yoshii, D., Extended affine Root System IV (Simply Laced Elliptic Lie Algebras), preprint (1999).

[Sh] Shimada, I., Picard-Lefschetz theory for the universal covering of complements to affine hypersurfaces, Publ. RIMS, Kyoto Univ., 32 (1996), 835-928.

[Si] Siersma, D., Classification and deformation of singularities, Proefschrift, Amsterdam, 1974.

[S11] Slodowy, P., Beyond Kac-Moody algebras and inside, Can. Mth. Soc. Proc., 5 (1986), 117-122.

[S12] - Singularitäten, Kac-Moody-Liealgebren, assoziierte Gruppen und Verallgemeinerungen, Habilitationsschrift, Bonn Univ., 1984.

[L] van der Lek, H., Extended Artin Groups, Proc. Symp. Pure Math., 40 Part 2 (1983), 117-121. 